\title{
IMPORTANCE OF MEAN BLOOD PRESSURE AND BLOOD PRESSURE VARIABILITY FOR THE EFFECT OF VALSARTAN VERSUS AMLODIPINE ON CARDIOVASCULAR EVENTS AND DEATH
}

Mehlum, M. Hollund ${ }^{\prime}$; Liest $\varnothing 1$, K. ${ }^{2}$; Kjeldsen, S.E. ${ }^{3}$ Julius, S. ${ }^{4}$ Hua, T.A. ${ }^{5}$; Rothwell, P.M. ${ }^{6}$;

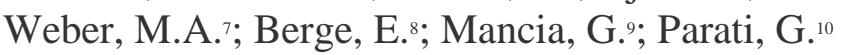

Author Information

Journal of Hypertension: June 2018 - Volume 36 - Issue - p e28-e29

doi: 10.1097/01.hjh.0000539038.77049.a3

Metrics

Abstract

\section{Objective:}

We explored if differences in blood pressure profiles can explain the differences in risks of cardiovascular events and death among patients treated with valsartan or amlodipine in the Valsartan Antihypertensive Long-Term Use Evaluation (VALUE) trial.

\section{Design and method:}

The VALUE trial was a randomised, double-masked trial of valsartan versus amlodipine in patients with hypertension. Mean follow-up was 4.2 years. We calculated mean systolic blood pressure as a time-dependent variable and blood pressure variability as the standard deviation (SD) of systolic blood pressure during follow-up in patients with 3 or more visits, and compared blood pressure profiles in the treatment groups. We performed multiple Cox regression analyses to assess the importance of mean blood pressure, blood pressure variability and clinical risk factors for the effects of valsartan versus amlodipine on myocardial infarction, stroke, congestive heart failure, and death.

\section{Results:}

14,996 patients were eligible for analysis. Mean systolic blood pressure and blood pressure variability was higher in the valsartan group (mean difference $2.2 \mathrm{~mm} \mathrm{Hg}$ and $1.4 \mathrm{~mm} \mathrm{Hg}, \mathrm{p}$ $<0.0001$ and $\mathrm{p}<0.0001$, respectively). For myocardial infarction, adjustment for mean blood pressure and blood pressure variability attenuated the risk increase for valsartan towards the null (from HR 1.19 to 1.12), mainly attributable to blood pressure variability. For stroke, adjustment for mean pressure and blood pressure variability both attenuated the risk increase for valsartan (from HR 1.12 to 1.01). For congestive heart failure the risk reduction for valsartan became even more pronounced (from HR 0.89 to 0.77 ). No clear effect was seen on death (from HR 1.01 to 1.00). The effects were the same if we excluded measurements from the first 6 months.

\section{Conclusions:}

Differences in mean systolic blood pressure and pressure variability during follow-up explained most of the effects of valsartan versus amlodipine on risk of myocardial infarction and stroke. Differences in variability seem to be particularly important for the effect on myocardial infarction. For congestive heart failure, there seems to be a beneficial effect of valsartan versus amlodipine, independent of blood pressure. These data deserve further investigation. 